\title{
44506 - PEDIATRIC CARDIAC SURGERY IN MORROCO: AN ANESTHETIC POINT OF VIEW
}

\author{
Geneviève Côté, CHUM Sainte-Justine, Montréal, QC, Canada; \\ Nancy Poirier, CHUM Sainte-Justine; \\ M Couture, CHUM Ste-Justine; \\ J Miro, CHUM Sainte-Justine;
}

\begin{abstract}
PURPOSE: The vast majority of Moroccan children with congenital cardiac disease remain without treatment because of lack of specialized resources and/or financial means to pay for interventions. A Canadian team of 12 physicians, technicians and nurses spent 2 weeks (March 2006) in Casablanca to help treat children while providing continuing education to the Moroccan pediatric cardiology/cardiac surgery community. We would like to portray the local limitations pertaining to surgery, anesthesia and post-operative care and follow up. Comparison between Canadian and Moroccan medical realities will be discussed.

CLINICAL FEATURES: During a 2 week period, 96 children were evaluated clinically and underwent an echocardiogram. Fifteen patients, two adults (41 and 32 years) and 13 children with a mean age of 5.7 years (range $=1$ month and 17.5 years) and mean weight of $19 \mathrm{~kg}$ (range $=2.8-60 \mathrm{Kg}$ ) underwent surgical correction of congenital cardiac anomalies. Surgical interventions were performed with cardiopulmonary bypass (CPB) in 11 patients including repairs of tetralogy of Fallot (4), sinus venosus and secundum atrial septal defect (2), mitral valve disease (2), aortic valve stenosis (1), partial atrio-ventricular canal defect (1), and a ventricular septal defect in the smallest CPB patient weighing $7.5 \mathrm{~kg}$. Four procedures were performed without CPB including 2 ductal ligations with pulmonary artery banding and a coarctation repair. The fourth off-pump procedure was an emergency pericardial drainage postcatherisation with dilation of the mitral valve performed with a per-auricular balloon, representing the first hybrid procedure in the country. There was no postoperative mortality and postoperative morbidity included reversible low cardiac syndrome in one patient and a pericardial effusion which was percutaneously drained. All patients were extubated in surgical suite. The mean intensive care stay of 2.1 days (range $=1-6$ days) and a mean hospital stay of 8 days (range $=6-11$ days). The cardiac catherisation team also performed 26 procedures with 16 interventions and 10 diagnostic studies. There was only 1 death following an interventional catherisation in an unstable 1 month old child in with pulmonary atresia and intact septum with a restrictive ASD and aortic stenosis. The overall mortality of the mission was $2.4 \%$ with excellent functional results.

CONCLUSION: Quality pediatric cardiac surgery and anesthesia is possible in developing countries with excellent results. Efforts should be continued to promote education of African teams to continue the treatment of children locally in their country.
\end{abstract}

